\title{
A Reliability Assessment Framework for Systems With Degradation Dependency by Combining Binary Decision Diagrams and Monte Carlo Simulation
}

\author{
Yan-Hui Lin, Yan-Fu Li, Senior Member, IEEE, and Enrico Zio, Senior Member, IEEE
}

\begin{abstract}
Components are often subject to multiple competing degradation processes. This paper presents a reliability assessment framework for multicomponent systems whose component degradation processes are modeled by multistate and physicsbased models with limited statistical degradation/failure data. The piecewise-deterministic Markov process modeling approach is employed to treat dependencies between the degradation processes within one component or/and among components. A computational method combining binary decision diagrams (BDDs) and Monte Carlo simulation (MCS) is developed to solve the model. A BDD is used to encode the fault tree of the system and obtain all the paths leading to system failure or operation. MCS is used to generate random realizations of the model and compute the system reliability. A case study is presented, with reference to one branch of the residual heat removal system of a nuclear power plant.
\end{abstract}

Index Terms-Binary decision diagrams (BDDs), degradation dependency, Monte Carlo simulation (MCS), piecewise-deterministic Markov process (PDMP), system reliability analysis.

\section{NOMENCLATURE}

$\begin{array}{ll}\text { Acronyms } & \\ \text { PBMs } & \text { Physics-based models. } \\ \text { MSMs } & \text { Multistate models. } \\ \text { FTA } & \text { Fault tree analysis. } \\ \text { CCFs } & \text { Common cause failures. } \\ \text { BDDs } & \text { Binary decision diagrams. } \\ \text { MCS } & \text { Monte Carlo simulation. } \\ \text { RHRS } & \text { Residual heat removal system. } \\ \text { WDFLM } & \text { Weighting depth-first left-most. } \\ \text { DFLM } & \text { Depth-first left-most. } \\ \text { ite } & \text { if-then-else. }\end{array}$

Manuscript received February 4, 2015; revised August 10, 2015; accepted October 5, 2015. This work was supported by the Electricité de France under Contract 8610-5910112096. This paper was recommended by Associate Editor L. Fang.

Y.-H. Lin and Y.-F. Li are with the Chair System Science and the Energy Challenge, Fondation Electricité de France, CentraleSupélec, Université Paris-Saclay, Châtenay-Malabry 92290, France (e-mail: yanhui.lin@centralesupelec.fr; yanfu.li@centralesupelec.fr).

E. Zio is with the Chair System Science and the Energy Challenge, Fondation Electricité de France, CentraleSupélec, Université Paris-Saclay, Châtenay-Malabry 92290, France, and also with the Politecnico di Milano, Milan 20133, Italy (e-mail: enrico.zio@centralesupelec.fr).

Color versions of one or more of the figures in this paper are available online at http://ieeexplore.ieee.org.

Digital Object Identifier 10.1109/TSMC.2015.2500020

\begin{tabular}{|c|c|}
\hline \multicolumn{2}{|l|}{ Notations } \\
\hline$C$ & Number of components in the system. \\
\hline$L$ & $\begin{array}{l}\text { Group of degradation processes modeled } \\
\text { by PBMs. }\end{array}$ \\
\hline $\boldsymbol{K}$ & $\begin{array}{l}\text { Group of degradation processes modeled } \\
\text { by MSMs. }\end{array}$ \\
\hline $\boldsymbol{D}_{O_{c}}$ & Degradation state of component $O_{c}$ \\
\hline $\overrightarrow{X_{L_{m}}}(t)$ & $\begin{array}{l}\text { Time-dependent continuous variables of } \\
\text { degradation process } L_{m} .\end{array}$ \\
\hline$X_{L_{m}}^{D}(t)$ & Nondecreasing degradation variables vector. \\
\hline $\overrightarrow{X_{L_{m}}^{P}}(t)$ & Physical variables vector. \\
\hline $\begin{array}{l}\mathcal{F}_{L_{m}} \\
Y_{K}(t)\end{array}$ & $\begin{array}{l}\text { Set of failure states of degradation process } L_{m} \text {. } \\
\text { State variable of degradation process } K_{m}\end{array}$ \\
\hline$S_{K_{n}}$ & Finite state set of degradation process $K_{n}$. \\
\hline $\mathcal{F}_{K_{n}}$ & Set of failure states of degradation process $K_{n}$. \\
\hline$\vec{Z}(t)$ & Degradation state of the system. \\
\hline & Environmental and operational factors in $K_{n}$. \\
\hline $\begin{array}{l}\lambda_{i}\left(j \mid \boldsymbol{\theta}_{K_{n}}\right) \\
\boldsymbol{\theta}_{I}\end{array}$ & $\begin{array}{l}\text { Transition rate from state } i \text { to } j \text {. } \\
\text { Environmental and operational factors in } L_{m} \text {. }\end{array}$ \\
\hline $\overrightarrow{f_{L_{m}}}\left(\cdot, \cdot \mid \boldsymbol{\theta}_{L_{m}}\right)$ & Physics equations of degradation process $L_{m}$. \\
\hline $\overrightarrow{Z_{p, q}}(t)$ & $\begin{array}{l}\text { Stochastic process of one group of interde- } \\
\text { pendent degradation processes. }\end{array}$ \\
\hline$N\left(\cdot, \cdot, \mid \boldsymbol{\theta}_{K_{n}}\right)$ & Semi-Markov kernel. \\
\hline
\end{tabular}

\section{INTRODUCTION}

M OST components undergo degradation processes before failure. A number of degradation models have been proposed in the field of reliability engineering based on the available information/data, which can be mainly classified into the following groups: 1) statistical distributions (e.g., Bernstein distribution [1]); 2) stochastic processes (e.g., Gamma process [2]); 3) MSMs (e.g., semi-Markov model [3]); and 4) PBMs (e.g., probabilistic superposition model [4]). Among the existing degradation models, PBMs [5]-[7] and MSMs [8]-[10] can be used to describe the evolution of degradation in structures, systems, and components, for which statistical degradation/failure data are insufficient, e.g., the highly reliable devices in the nuclear and aerospace industries. A PBM gives an integrated mechanistic description of the component life consistent with the 
underlying real degradation mechanisms (e.g., wear, corrosion, fatigue, etc.) by using physics knowledge and equations [4], whereas an MSM describes the degradation process in a discrete way, supported by material science knowledge, degradation and/or failure data from historical field collection or degradation tests [11], [12].

In reality, components are often subject to multiple competing degradation processes. The dependencies among these processes within one component (e.g., the wear of rubbing surfaces influenced by the environmental stress shock within a micro-engine [13]), or/and among different components (e.g., the degradation of the prefiltrations stations leading to a lower performance level of the sand filter in a water treatment plant [14]) need to be considered. Components can be dependent due to functional dependence, where the failure of a trigger component causes other components to become inaccessible or unusable [15], [16]. Competing failure propagation and failure isolation effects have been studied in [17] and [18], where a failure not only causes outage to the component from which the failure originates, but also propagates through all other system components causing the entire system failure and failure isolation occurs when the failure of one component causes other components within the same system to become isolated from the system.

Recently, Lin et al. [19] employed the piecewisedeterministic Markov process (PDMP) modeling framework to integrate PBMs and MSMs for treating the dependencies among degradation processes for a system with a small number of components, where the whole system is modeled by one PDMP. For systems of larger size, the high dimension of its PDMP can lead to very heavy computational burdens, because solving the PDMP of a small system is already time consuming due to the combinatorial nature of MSMs and the need to simulate the trajectory between any two system states [19]. In addition, the dependencies may only exist within certain groups of components and leave different groups being independent [20], and the causes of systems failure are not easy to be identified.

FTA [21] is typically used to identify the combinations of events leading to system failure and compute its probability by using minimal cut sets found from the fault tree structure. For real systems, this can be computationally intensive, when the tree structure is large and, especially, if it contains repeated basic events [22]. In addition, all basic events are usually assumed statistically independent.

CCFs of components have been considered in [23]-[25]: implicit and explicit methods have been developed to evaluate the system reliability. In binary-state systems, components failures with dependent propagation effects have been studied in [26], within a dynamic FTA framework. The statistical dependence of component states across different phases of phased-mission systems has been treated by using multiple-valued decision diagrams to encode fault trees in [27] and [28].

On the contrary, the dependencies of the degradation processes leading to failure of different components need to be considered which render certain basic events under different gates being dependent. To the knowledge of Song et al. [29], there is no published research work to tackle this problem, of practical reference.

To take into account such dependencies at a relatively low computational cost for systems of larger size, a system reliability assessment method is proposed combining BDDs [30] and MCS [31]. Instead of modeling the degradation of the whole system by one PDMP as in [19], the proposed method can identify the groups of components being dependent and decompose the original PDMP into a group of smaller ones which are independent from each other and easier to be solved. Besides, the states of these PDMPs leading to the systems failure can be easily obtained. First, a fault tree is transformed to a BDD from which all paths leading to the system failure or operation can be efficiently obtained. BDDs [30] are directed acyclic graphs, encoding Shannon's decomposition of a formula, and have been implemented in many domains; they possess the feature of sharing equivalent subgraphs and hence can reduce the computational time and memory requirements [32]. An algorithm based on BDD has been developed for reliability analysis of phased-mission systems with multimode failures in [33] to improve the efficiency and reduce the computational complexity. BDD has also been employed for network reliability and sensitivity analysis in [34]. Second, MCS is used to estimate the probability of each path to compute the system reliability taking into account the dependencies between basic events, since analytically solving the PDMPs is difficult, if not impossible, due to the large size and complex behavior of the system [35].

The rest of this paper is organized as follows. Section II provides the assumptions and model descriptions. The proposed reliability assessment method is presented in Section III. Section IV presents one case study on one branch of a RHRS of a nuclear power plant. Section V concludes the paper.

\section{Assumption AND Model Description}

\section{A. General Assumptions}

We consider a multicomponent system, made of $C$ components denoted by $\boldsymbol{O}=\left\{O_{1}, O_{2}, \ldots, O_{C}\right\}$.

The following assumptions are made.

1) The fault tree of the system is available and contains $Q$ basic events denoted by $\boldsymbol{e}=\left\{e_{1}, e_{2}, \ldots, e_{Q}\right\}$ which include the failures of components and other events such as erroneous operation caused by human errors. The component-failure type of events are determined by their underlying degradation processes.

2) Each component may be affected by multiple degradation processes, possibly dependent. The degradation processes can be separated into two groups: a) $\boldsymbol{L}=$ $\left\{L_{1}, L_{2}, \ldots, L_{M}\right\}$ modeled by $M$ PBMs and b) $\boldsymbol{K}=$ $\left\{K_{1}, K_{2}, \ldots, K_{N}\right\}$ modeled by $N$ MSMs, where $L_{m}$, $m=1,2, \ldots, M$ and $K_{n}, n=1,2, \ldots, N$ are the indexes of the degradation processes. The degradation state of a component $O_{c} \in \boldsymbol{O}, c=1,2, \ldots, C$, is determined by its degradation processes $\boldsymbol{D}_{O_{c}} \subseteq \boldsymbol{L} \cup \boldsymbol{K}$ and the component fails when its degradation processes enter its failure state space (see items 3 and 4 below for its definition). 


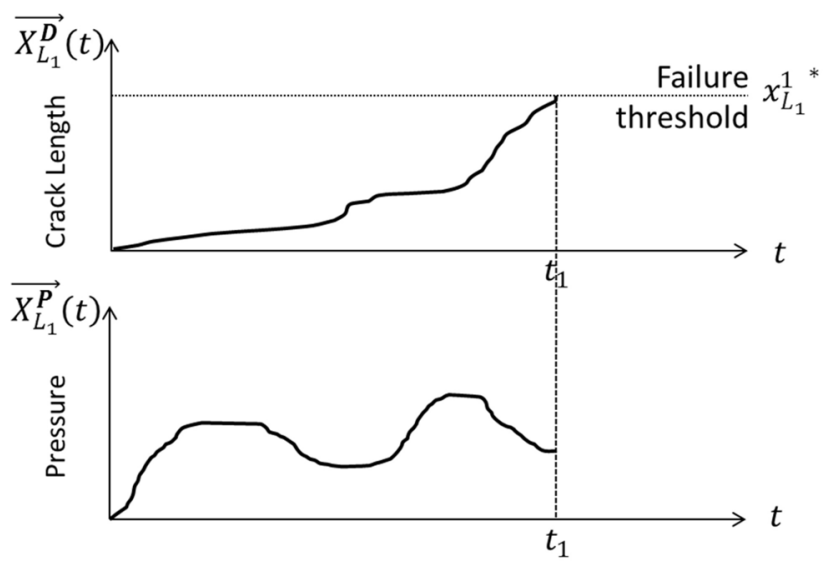

Fig. 1. Illustration of $L_{1}$.

3) A degradation process $L_{m} \in \boldsymbol{L}$ in the first group is described by $d_{L_{m}}$ time-dependent continuous variables $\overrightarrow{X_{L_{m}}}(t)=\left(\overrightarrow{X_{L_{m}}^{\boldsymbol{D}}}(t), \overrightarrow{X_{L_{m}}^{\boldsymbol{P}}}(t)\right) \in \mathbb{R}^{d_{L_{m}}}$ in terms of: a) the nondecreasing degradation variables vector $\overrightarrow{X_{L_{m}}^{D}}(t)$ (e.g., crack length) representing the component degradation condition and b) the physical variables $\overrightarrow{X_{L_{m}}^{P}}(t)$ (e.g., velocity) influencing $\overrightarrow{X_{L_{m}}^{D}}(t)$ and vice versa. $d_{L_{m}}$ is the number of nondecreasing degradation variables and physical variables for a degradation process $L_{m}$. Their evolution is characterized by a system of first-order differential equations $\overrightarrow{{X_{L_{m}}}}(t)=\overrightarrow{f_{L_{m}}}\left(\overrightarrow{X_{L_{m}}}(t), t \mid \boldsymbol{\theta}_{L_{m}}\right)$, i.e., physics equations, where $\boldsymbol{\theta}_{L_{m}}$ represents the environmental factors to $L_{m}$ (e.g., temperature and pressure) and the parameters used in $\overrightarrow{f_{L_{m}}}$. The evolution of physical variables can be characterized by physics equations. The environmental factors are the parameters of the physics equations and their evolution is not characterized by physics equations. If any environmental or operational factor is modeled by physics equations and influencing the degradation variables, then, it is considered as one physical variable. $L_{m}$ fails when one $x_{L_{m}}^{i}(t) \in \overrightarrow{X_{L_{m}}^{D}}(t)$ reaches or exceeds its corresponding failure threshold denoted by $x_{L_{m}}^{i}{ }^{*}$. The failure state set of $L_{m}$ is denoted by $\mathcal{F}_{L_{m}}$. An example of $L_{1}$ is shown in Fig. 1 .

4) A degradation process $K_{n} \in \boldsymbol{K}$ in the second group is described by the state variable $Y_{K_{n}}(t)$, which takes values from a finite state set $\boldsymbol{S}_{K_{n}}=\left\{0_{K_{n}}, 1_{K_{n}}, \ldots, d_{K_{n}}\right\}$, where " $d_{K_{n}}$ " is the perfect functioning state and " $0_{K_{n}}$ " is the complete failure state. All intermediate states are functioning or partially functioning. The transition rates $\lambda_{i}\left(j \mid \boldsymbol{\theta}_{K_{n}}\right), \forall i, j \in S_{K_{n}}, i>j$ characterize the degradation transition probabilities from state $i$ to state $j$, where $\boldsymbol{\theta}_{K_{n}}$ represents the environmental factors to $K_{n}$ and the related coefficients of $\lambda_{K_{n}}$. The failure state set of $K_{n}$ is denoted by $\mathcal{F}_{K_{n}}=\left\{0_{K_{n}}\right\}$. An example of $K_{1}$ is shown in Fig. 2.

Dependencies between degradation processes may exist both within and across groups $\boldsymbol{L}$ and $\boldsymbol{K}$. The degradation levels of the components in the first group may influence the

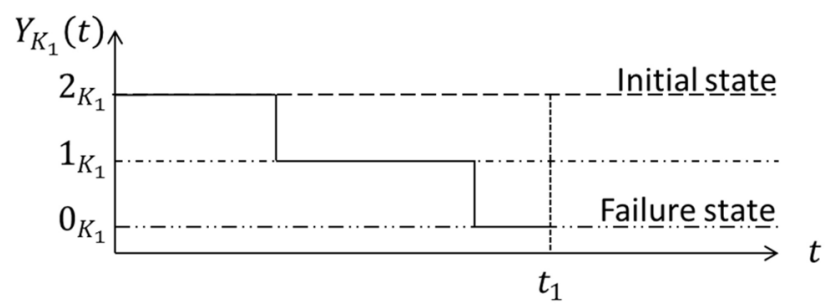

Fig. 2. Illustration of $K_{1}$.

transition times and transition directions of the degradation processes of the second group and the degradation states of the second group may influence the evolution trajectories of the continuous variables in the first group [19]. PDMPs are employed to model this dependency, the detailed formulations are shown in (1) and (2).

\section{B. PDMPs for Dependent Degradation Processes}

Let us consider one group of interdependent degradation processes $\boldsymbol{L}_{p}=\left\{L_{p_{1}}, \ldots, L_{p_{n}}\right\}$ and $\boldsymbol{K}_{q}=\left\{K_{q_{1}}, \ldots, K_{q_{m}}\right\}$, which have no dependencies with the other degradation processes. Their degradation states are represented by

$$
\begin{aligned}
& \overrightarrow{Z_{p, q}}(t)=\left(\begin{array}{c}
\left(\begin{array}{c}
\overrightarrow{X_{L_{p_{1}}}}(t) \\
\vdots \\
\overrightarrow{X_{L_{p}}}(t)
\end{array}\right)=\vec{X}_{p}(t) \\
\left(\begin{array}{c}
Y_{q_{1}}(t) \\
\vdots \\
Y_{q_{m}}(t)
\end{array}\right)=\vec{Y}_{q}(t)
\end{array}\right) \\
& \in \boldsymbol{E}_{p, q}=\mathbb{R}^{d_{\mathrm{L}_{p}}} \times \boldsymbol{S}_{\boldsymbol{K}_{q}}, \quad \forall t \geq 0
\end{aligned}
$$

where $\boldsymbol{E}_{p, q}$ is the space combining $\mathbb{R}^{d_{\boldsymbol{L}_{p}}}\left(d_{\boldsymbol{L}_{p}}=\sum_{k=1}^{n} d_{L_{p_{k}}}\right)$ and $\boldsymbol{S}_{\boldsymbol{K}_{q}}=\left\{0,1, \ldots, d_{\boldsymbol{K}_{q}}\right\}$ denotes the state set of process $\vec{Y}_{q}(t)$.

The evolution of the vector of degradation states $\overrightarrow{Z_{p, q}}(t)$ involves: 1) the stochastic transition process of $\vec{Y}_{q}(t)$ and 2) the deterministic progression of $\vec{X}_{p}(t)$, between successive transitions of $\vec{Y}_{q}(t)$, given $\vec{Y}_{q}(t)$. The first process is governed by the transition rates of $\vec{Y}_{q}(t)$, which depend on the degradation levels of the components in the first group, as follows:

$$
\begin{aligned}
\lim _{\Delta t \rightarrow 0} \quad P\left(\vec{Y}_{q}(t+\Delta t)=\vec{j} \mid \overrightarrow{Z_{p, q}}(t)\right. \\
\left.=\left(\vec{X}_{p}(t), \vec{Y}_{q}(t)=\vec{i}\right)^{T}, \boldsymbol{\theta}_{\boldsymbol{K}_{q}}\right) \\
=\lambda_{\vec{i}}^{q}\left(\vec{j} \mid \vec{X}_{p}(t), \boldsymbol{\theta}_{\boldsymbol{K}_{q}}\right) \Delta t, \quad \forall \vec{i}, \vec{j} \in \boldsymbol{S}_{\boldsymbol{K}_{q}}, \vec{i} \neq \vec{j}
\end{aligned}
$$

where the parameter vector $\boldsymbol{\theta}_{\boldsymbol{K}_{q}}$ represents environmental and operational factors influencing the degradation processes in $\boldsymbol{K}_{q}$. The second evolution process is described by the deterministic physics equations which depend on the degradation 
states of the second group as follows:

$$
\begin{aligned}
\overrightarrow{\dot{X}_{p}}(t)= & \left(\begin{array}{c}
\overrightarrow{X_{L_{p_{1}}}}(t) \\
\vdots \\
\overrightarrow{X_{L_{p_{n}}}}(t)
\end{array}\right)=\left(\begin{array}{c}
\overrightarrow{f_{L_{p_{1}}}}\left(\overrightarrow{Z_{p, q}}(t), t \mid \boldsymbol{\theta}_{L_{p_{1}}}\right) \\
\vdots \\
\overrightarrow{f_{L_{p_{n}}}}\left(\overrightarrow{Z_{p, q}}(t), t \mid \boldsymbol{\theta}_{L_{p_{n}}}\right)
\end{array}\right) \\
= & \overrightarrow{f_{\boldsymbol{L}_{p}}}\left(\overrightarrow{Z_{p, q}}(t), t \mid \boldsymbol{\theta}_{\boldsymbol{L}_{p}}=\left(\boldsymbol{\theta}_{L_{p_{1}}}, \ldots, \boldsymbol{\theta}_{L_{p_{n}}}\right)\right)
\end{aligned}
$$

where the parameter vector $\boldsymbol{\theta}_{L_{p_{k}}}, k=1,2, \ldots, n$ represents environmental and operational factors influencing the degradation processes in $L_{p_{k}}$. It should be noted that the evolution of one degradation process in $\overrightarrow{Z_{p, q}}(t)$ depends on the states of all the degradation processes in $\overrightarrow{Z_{p, q}}(t)$.

\section{Methodology}

In this section, a computational method combining BDDs and MCS is proposed.

\section{A. $B D D s$}

A BDD is a directed acyclic graph encoding Shannon's decomposition of a formula. A BDD has two terminal vertices labeled 1 and 0 to indicate the failure and operation of the system, respectively. Each nonterminal vertex is labeled with a variable and has two outgoing edges: 1) 1-edge and 2) 0-edge which indicate the occurrence and nonoccurrence of the corresponding basic event, respectively.

A BDD is employed to encode the fault tree of the system according to the given ordering of the indicator variable $X_{i}$ which denotes the occurrence or nonoccurrence of the basic event $i$ ( $X_{i}=1$ indicating the occurrence of the basic event $i$ and $X_{i}=0$ indicating the opposite). The size of the BDD largely depends on the given ordering and the problem of finding the global optimal ordering is an intractable task [36], [37]. Several ordering heuristics have been developed, whose performances may vary on different problems. In this paper, we employ the WDFLM ordering technique proposed in [38], which leads to satisfactory results according to the tests in [39] and [40]. WDFLM first assigns weight 1 to each basic event. Then, it traverses the fault tree bottom-up to calculate the weight of each gate by adding the weights of all its inputs, i.e., gates and basic events. Fig. 3 shows an example of a fault tree where the weights of the gates are obtained through WDFLM. Then, the inputs of a gate are rearranged in the order of increasing weights as shown in Fig. 4.

Finally, the DFLM ordering technique [41] is applied to the fault tree to get the variable ordering. In this technique, the basic events are placed in the ordered list as soon as they are encountered during the DFLM traversal of the fault tree. Let $<$ be a total ordering of variables, for the fault tree in Fig. 3, it is $X_{3}<X_{4}<X_{1}<X_{2}$.

Based on the variable ordering, the related BDD can be constructed using the bottom-up procedure. First, all basic events $i, i \in \boldsymbol{e}$ are associated with the ite structure [42] ite $\left(X_{i}, 1,0\right)$, where ite $\left(X_{i}, f_{1}, f_{2}\right)=\left(X_{i} \wedge f_{1}\right) \vee\left(\neg X_{i} \wedge f_{2}\right)$ which means if the basic event $i$ occurs then consider function $f_{1}$ else consider function $f_{2}$. Then, work from the bottom to the top of the fault tree and obtain the ite structure for each gate by using

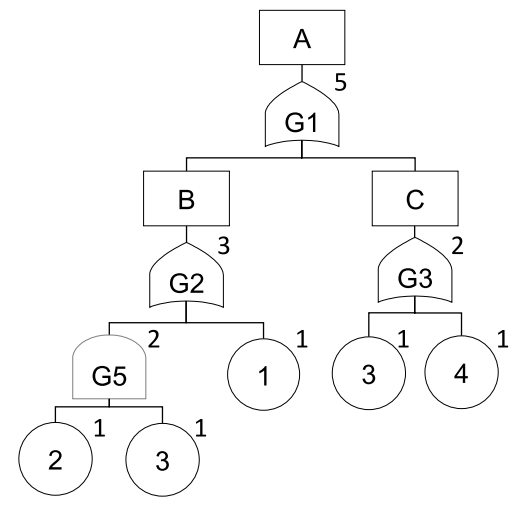

Fig. 3. Illustration of fault tree labeled with weights.

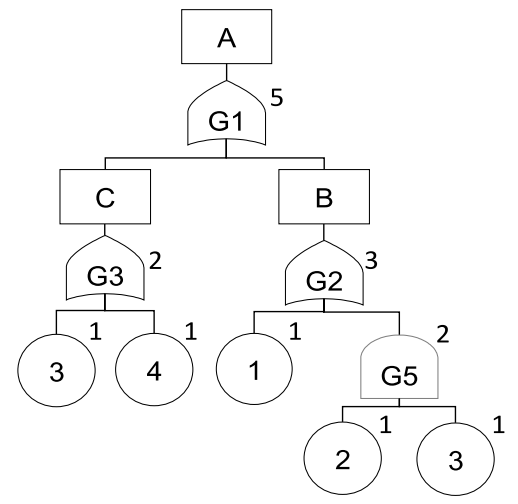

Fig. 4. Illustration of fault tree with rearranged inputs of gates.

the following principle: let us consider two variables $X_{a}<X_{b}$ and four functions $f_{1}-f_{4}$, let $<>$ be any logic operation AND or OR, then

$\operatorname{ite}\left(X_{a}, f_{1}, f_{2}\right)<>\operatorname{ite}\left(X_{a}, f_{3}, f_{4}\right)=\operatorname{ite}\left(X_{a}, f_{1}<>f_{3}, f_{2}<>f_{4}\right)$

and

$$
\begin{aligned}
& \operatorname{ite}\left(X_{a}, f_{1}, f_{2}\right)<>\operatorname{ite}\left(X_{b}, f_{3}, f_{4}\right) \\
& \quad=\operatorname{ite}\left(X_{a}, f_{1}<>\operatorname{ite}\left(X_{b}, f_{3}, f_{4}\right), f_{2}<>\operatorname{ite}\left(X_{b}, f_{3}, f_{4}\right)\right) .
\end{aligned}
$$

The ite structure of the top event of the fault tree in Fig. 3 can be obtained as ite $\left(X_{3}, 1\right.$, ite $\left(X_{4}, 1\right.$, ite $\left.\left.\left(X_{1}, 1,0\right)\right)\right)$. The associated BDD shown in Fig. 5 can be constructed by breaking down each ite structure into its left and right branches, and eliminating the vertexes that are not useful (a vertex is not useful when its two outgoing edges point to the same vertex or it is equivalent to another vertex) [43].

Finally, all the paths leading to system failure can be obtained as: 1) $X_{3}=1$; 2) $X_{3}=0, X_{4}=1$; and 3) $X_{3}=0$, $X_{4}=0, X_{1}=1$ and the path leading to system operation is $X_{3}=0, X_{4}=0$, and $X_{1}=0$. The exact system reliability is equal to the sum of the probability of occurrence of each path leading to system operation or 1-the sum of the probability of occurrence of each path leading to system failure.

\section{B. MCS for PDMPS}

To derive the probability of occurrence of one path, all the PDMPs containing the variables involved in that path need to 


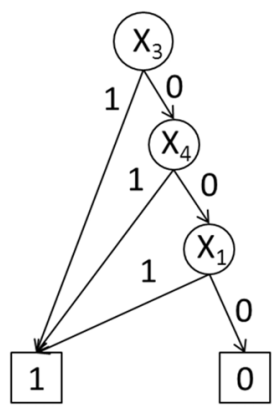

Fig. 5. BDD for fault tree in Fig. 3.

be solved. Since the PDMPs are independent from each other, the product of the probabilities of PDMPs being in the states indicated by the path equals the probability of occurrence of that path. Analytically solving the PDMPs is a difficult task, whereas MCS is well suited.

We develop a MCS algorithm for solving the PDMPs. It consists of sampling the transition time and the arrival state for the MSMs and, then, calculating the behavior of the PBMs within the transition times using the physics equation.

Refer to one PDMP presented in Section II-A. Let $\overrightarrow{Z_{p, q}^{k}}=$ $\overrightarrow{Z_{p, q}}\left(T^{k}\right)=\left(\begin{array}{c}\overrightarrow{X_{p}}\left(T^{k}\right) \\ \overrightarrow{Y_{q}^{k}}\end{array}\right) \in \boldsymbol{E}_{p, q}, k \in \mathbb{N}$, where $\overrightarrow{Y_{q}^{k}} \in S_{K_{q}}, k \in \mathbb{N}$ denotes the state of $\vec{Y}_{q}(t)$ after $k$ transitions from the beginning (a transition occurs as long as any one of the elements in $\vec{Y}_{q}(t)$ changes its state) and $T^{k}$ denotes the time of arrival at state $\overrightarrow{Y_{q}^{k}}$. Then, $\left\{\overrightarrow{Z_{p, q}^{k}}, T^{k}\right\}_{k \geq 0}$ is a Markov renewal process defined on the space $\boldsymbol{E}_{p, q} \times \mathbb{R}^{+}$[44]. We can obtain that

$$
\begin{gathered}
P\left[\overrightarrow{Z_{p, q}^{n+1}} \in B, T^{n+1} \in\left[T^{n}, T^{n}+\Delta t\right] \mid \overrightarrow{Z_{p, q}^{n}}=\vec{i}, \boldsymbol{\theta}_{K_{q}}\right] \\
=\iint_{B *[0, \Delta t]} N\left(\vec{i}, \overrightarrow{d z}, d s \mid \boldsymbol{\theta}_{K_{q}}\right), \\
\forall n \geq 0, \Delta t \geq 0, \vec{i} \in \boldsymbol{E}_{p, q}, B \in \varepsilon
\end{gathered}
$$

where $\varepsilon$ is a $\sigma$-algebra of $\boldsymbol{E}_{p, q}$ and $N\left(\vec{i}, \overrightarrow{d z}, d s \mid \boldsymbol{\theta}_{K_{q}}\right)$ is a semi-Markov kernel on $\boldsymbol{E}_{p, q}$, which verifies that $\iint_{\boldsymbol{E}_{p, q} *[0, \Delta t]} N\left(\vec{i}, \overrightarrow{d z}, d s \mid \boldsymbol{\theta}_{K_{q}}\right) \leq 1, \forall \Delta t \geq 0, \vec{i} \in \boldsymbol{E}_{p, q}$. It can be further developed as

$$
N\left(\vec{i}, \overrightarrow{d z}, d s \mid \boldsymbol{\theta}_{K_{q}}\right)=d F_{i}\left(s \mid \boldsymbol{\theta}_{K_{q}}\right) \beta\left(\vec{i}, \overrightarrow{d z} \mid s, \boldsymbol{\theta}_{K_{q}}\right)
$$

where

$$
d F_{\vec{i}}\left(s \mid \boldsymbol{\theta}_{K_{q}}\right)
$$

is the probability density function of $T^{n+1}-T^{n}$ given $\overrightarrow{Z_{p, q}^{n}}=\vec{i}$ and

$$
\beta\left(\vec{i}, \overrightarrow{d z} \mid s, \boldsymbol{\theta}_{K_{q}}\right)
$$

is the conditional probability of state $\overrightarrow{Z_{p, q}^{n+1}}$ given $T^{n+1}-T^{n}=s$.

\begin{tabular}{|c|}
\hline Determine the ordering of variables through WDFLM. \\
\hline Construct the BDD encoding the fault tree of the system. \\
\hline Obtain all the paths leading to system failure or operation. \\
\hline Divide the degradation processes involved in the paths into groups \\
that are independent from each other. \\
\hline Select the PDMPs for each group of the degradation processes. \\
\hline $\begin{array}{c}\text { Calculate the probabilities of PDMPs being in the states indicated by } \\
\text { the paths through MCS. }\end{array}$ \\
\begin{tabular}{|c|} 
Calculate the probability of occurrence of each path by multiplying the \\
probabilities of PDMPs being in the states indicated by the path.
\end{tabular} \\
$\begin{array}{c}\text { Calculate the system reliability by summing the probabilities of } \\
\text { occurrence of the paths leading to system operation or 1- the sum of } \\
\text { the probabilities of occurrence of the paths leading to system failure. }\end{array}$ \\
\hline
\end{tabular}

Fig. 6. Flowchart of the computational method.

The simulation procedure consists of sampling the transition time from (8) and the arrival state from (9) for $\vec{Y}_{q}(t)$, then, calculating $\vec{X}_{p}(t)$ within the transition times, by using the physics equation (3) until the time of system evolution reaches a certain mission time $T_{\text {miss }}$.

To calculate the probability of occurrence of one path (let $\overrightarrow{Z_{p, q}^{*}}$ indicate the state space, which contains all the states of $\overrightarrow{Z_{p, q}}(t)$ that are consistent with the state of the path), the procedure of the MCS is presented as follows.

Set $N_{\max }$ (the maximum number of replications) and $k=0$ (index of replication)

Set $k^{\prime}=0$ (number of trials that end in the state indicated by the path)

While $k<N_{\max }$

Initialize the system by setting $\overrightarrow{Z_{p, q}^{\prime}}(0)=\left(\begin{array}{c}\overrightarrow{X_{p}}(0) \\ \overrightarrow{Y_{q}^{\prime}}\end{array}\right)$ (initial state), and the time $T=0$ (initial system time)

Set $t^{\prime}=0$ (state holding time)

While $T \leq T_{\text {miss }}$

Sample a $t^{\prime}$ by using (8)

Sample an arrival state $\vec{Y}_{q}^{\prime \prime}$ for stochastic process $\vec{Y}_{q}(t)$ from all the possible states by using (9)

Calculate $\vec{X}_{p}(s), \forall s \in\left[T, T+t^{\prime}\right]$ by using eq. (3)

Set $\overrightarrow{Z_{p, q}^{\prime}}(s)=\left(\begin{array}{c}\overrightarrow{X_{p}}(s) \\ \overrightarrow{Y_{q}^{\prime}}\end{array}\right), \forall s \in\left[T, T+t^{\prime}[\right.$

Set $T=T+t^{\prime}, \overrightarrow{Z_{p, q}^{\prime}}(T)=\left(\begin{array}{c}\overrightarrow{X_{p}}(T) \\ \overrightarrow{Y_{q}^{\prime \prime}}\end{array}\right)$ and $\overrightarrow{Y_{q}^{\prime}}=\overrightarrow{Y_{q}^{\prime \prime}}$

End While

If $\overrightarrow{Z_{p, q}^{\prime}}\left(T_{\text {miss }}\right) \in \overrightarrow{Z_{p, q}^{*}}$

Set $k^{\prime}=k^{\prime}+1$

\section{End if}

Set $k=k+1$

\section{End While $\square$}

The estimated probability of occurrence of one path at time $T_{\text {miss }}$ can be obtained by

$$
\hat{P}\left(T_{\text {miss }}\right)=1-k^{\prime} / N_{\max }
$$




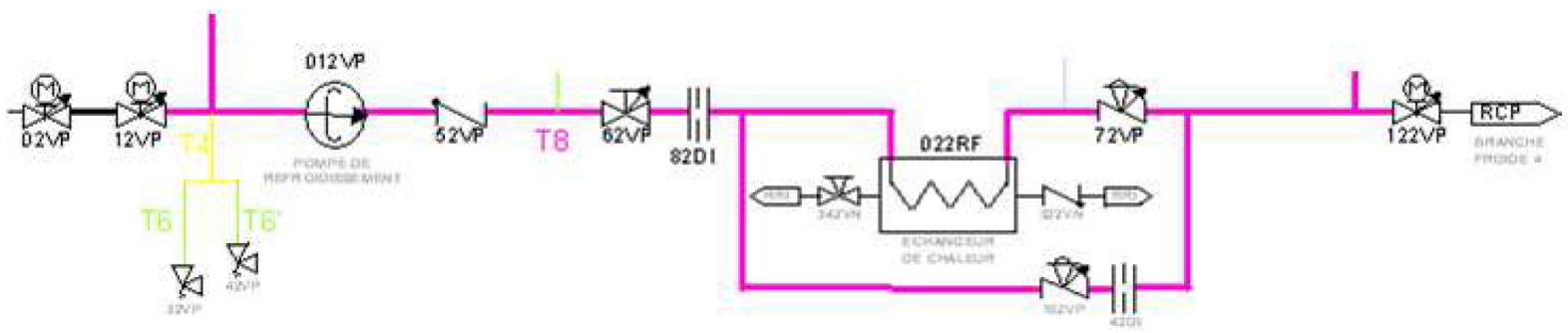

Fig. 7. Diagram of one branch of the RHRS.

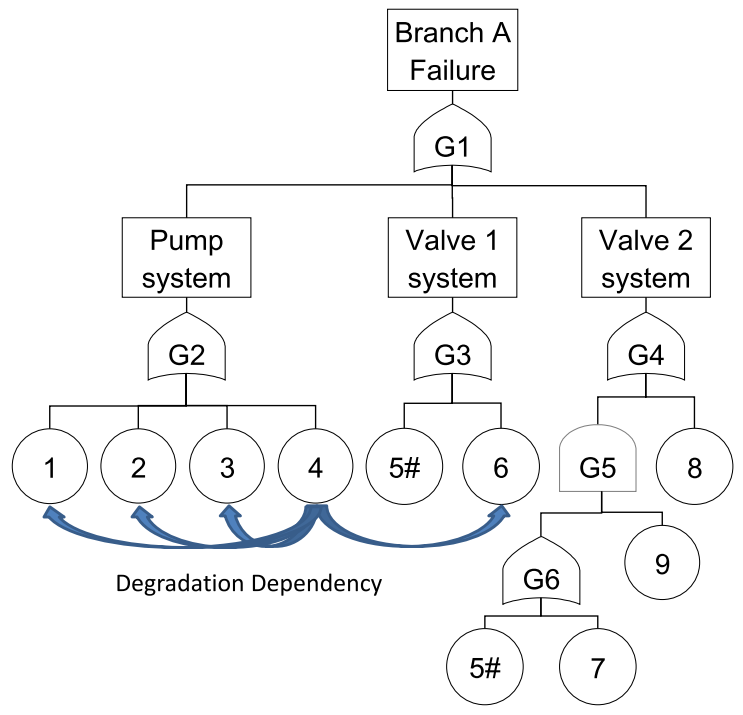

Fig. 8. Fault tree of one branch of the RHRS.

TABLE I

Definitions of THE BASIC Events

\begin{tabular}{ll}
\hline \hline \multicolumn{1}{c}{ Basic Event } & \multicolumn{1}{c}{ Definition } \\
\hline 1 & Failure of the circuit breaker \\
2 & Failure of the motor \\
3 & Failure of the pump contactor \\
4 & Failure of the pump \\
$5 \#$ & Closure due to human error \\
6 & Failure of the valve \\
7 & Failure of the diaphragm \\
8 & Failure of the pneumatic valve VP1 \\
9 & Failure of the pneumatic valve VP2 \\
\hline \hline
\end{tabular}

with the sample variance [45] as follows:

$$
\operatorname{var}_{\hat{P}\left(T_{\text {miss }}\right)}=\hat{P}\left(T_{\text {miss }}\right)\left(1-\hat{P}\left(T_{\text {miss }}\right)\right) /\left(N_{\text {max }}-1\right) .
$$

\section{MCS for PDMPs}

The flowchart of the whole proposed computational method combining BDDs and MCS is shown in Fig. 6.

\section{CASE STUdy}

The illustrative case refers to one branch of the RHRS [46] of a nuclear power plant shown in Fig. 7. The fault tree is shown in Fig. 8. The definitions of the basic events are presented in Table I.

By knowledge and experience of the field experts, the degradation dependency is described as follows: the degradation of
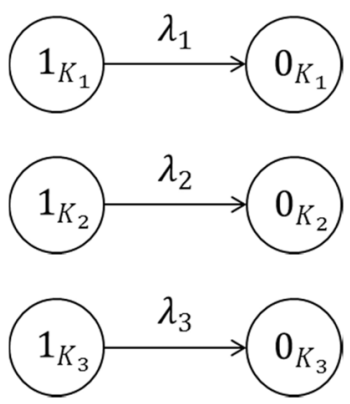

Fig. 9. Representation of the degradation processes of the circuit breaker, motor, and pump contactor.

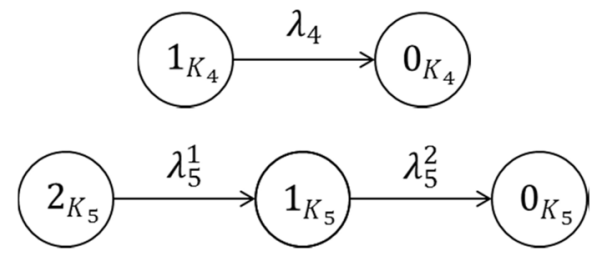

Fig. 10. Representation of the degradation processes of the pump.

the pump can lead it to vibrate [47], which will, in turn, cause the vibration of the other neighboring components (e.g., the valve) and therefore aggravate the degradation process of the latter [48]. The dependency exists between basic events 1-4 and 6, as indicated in Fig. 6. The component degradation models provided by the expert colleagues of Electricité de France are presented below. Some degradation processes are modeled by PBMs if their degradation data is unavailable and, thus, the physics equations have to be used, whereas the others are modeled by MSMs supported by the degradation and/or failure data from historical field collection.

The circuit breaker, motor, and pump contactor each have one degradation process modeled by MSMs $K_{1}-K_{3}$, respectively, as shown in Fig. 9.

The pump has two degradation processes modeled by MSMs $K_{4}$ and $K_{5}$, as shown in Fig. $10 . K_{4}$ relates to the failure on demand and $K_{5}$ relates to the external leakage which can cause the pump to vibrate when $Y_{K_{5}}(t)$ reaches the state $1_{K_{5}}$.

Closure due to human error follows one MSM $K_{6}$, as shown in Fig. 11.

The valve has one degradation process modeled by one PBM $L_{1}$ related to the crack propagation due to manufacturing defects. $L_{1}$ is based on a deterministic crack growth model, which follows Paris-Erdogan law [49]. For the phase of crack 


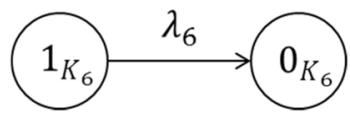

Fig. 11. Process of closure due to human error.



Fig. 12. Representation of the degradation processes of the pneumatic valves.

propagation, the threshold is defined as the number of cycles calculated as follows:

$$
N_{c}=\frac{1 /\left(\frac{m}{2}-1\right) *\left(1 / a_{0}^{\left(\frac{m}{2}-1\right)}-1 / a_{c}^{\left(\frac{m}{2}-1\right)}\right)}{C\left(f(R)_{\operatorname{Max}} Y_{\operatorname{Max}} \sqrt{\pi} \Delta \sigma_{\operatorname{Max}}\right)^{m}}
$$

where the definition of the parameters can be found in [50]. The valve fails when the number of solicitation exceeds $N_{c}$. The equivalent number of solicitations executed per year is assumed to be constant and equal to $d_{c}$.

The diaphragm has one degradation process modeled by one PBM $L_{2}$ related to the cavitation erosion mechanism, which can cause the thickness loss. The threshold is defined as the thickness required to ensure pressure resistance, which is calculated as follows:

$$
t_{m}=P D_{0} / 2(S+y P)
$$

where $P$ is the estimated pressure for RHRS, $D_{0}$ is the outside diameter of the pipe, $y$ is a coefficient, and $S$ is the allowable stress in the pipe. The diaphragm fails when the thickness loss exceeds $t_{m}$. The annual loss of thickness is assumed to be constant and equal to $d_{m}$.

The pneumatic valves VP1 and VP2 each have one degradation process modeled by MSMs $K_{7}$ and $K_{8}$, respectively, as shown in Fig. 12.

$K_{5}$ has impacts on $K_{1}-K_{4}$ and $L_{1}$. When $Y_{K_{5}}(t)$ reaches the state $1_{K_{5}}$ the transition rates of $K_{1}-K_{4}$ will increase to $\lambda_{1}^{\prime}, \lambda_{2}^{\prime}, \lambda_{3}^{\prime}$, and $\lambda_{4}^{\prime}$, respectively, and $d_{c}$ in $L_{1}$ will change to $d_{c}^{\prime}$. All the parameter values in the degradation models are presented in Table II. For confidentiality, we use artificially scaled values; they are set in a way to simulate the system under accelerated aging conditions.

Applying the WDFLM ordering heuristic [38], the variable ordering obtained is $X_{5 \#}<X_{6}<X_{1}<X_{2}<X_{3}<X_{4}<X_{8}<$ $X_{9}<X_{7}$. The corresponding BDD is shown in Fig. 13. There are two paths leading to system operation: 1) $X_{5 \#}=0, X_{6}=0$, $X_{1}=0, X_{2}=0, X_{3}=0, X_{4}=0, X_{8}=0, X_{9}=0$ and 2) $X_{5 \#}=0, X_{6}=0, X_{1}=0, X_{2}=0, X_{3}=0, X_{4}=0, X_{8}=0$, $X_{9}=1, X_{7}=0$.

The degradation processes are divided into five groups: 1) $\left\{K_{6}\right\}$;2) $\left\{L_{2}\right\}$; 3) $\left\{K_{7}\right\}$; 4) $\left\{K_{8}\right\}$; and 5) $\left\{K_{1}, K_{2}\right.$, $\left.K_{3}, K_{4}, K_{5}, L_{1}\right\}$. Each of the first four groups has only one
TABLE II

PARAMETER VAlues

\begin{tabular}{ll}
\hline \hline \multicolumn{1}{c}{ Parameter } & \multicolumn{1}{c}{ Value } \\
\hline$\lambda_{1}$ & $6.65 \mathrm{e}-8 / \mathrm{h}$ \\
$\lambda_{2}$ & $1.8 \mathrm{e}-6 / \mathrm{h}$ \\
$\lambda_{3}$ & $4.4 \mathrm{e}-7 / \mathrm{h}$ \\
$\lambda_{4}$ & $1.3 \mathrm{e}-5 / \mathrm{h}$ \\
$\lambda_{5}^{1}$ & $4.7 \mathrm{e}-5 / \mathrm{h}$ \\
$\lambda_{5}^{2}$ & $1.3 \mathrm{e}-5 / \mathrm{h}$ \\
$\lambda_{6}$ & $1.5 \mathrm{e}-5 / \mathrm{h}$ \\
$\lambda_{7}$ & $1.95 \mathrm{e}-8 / \mathrm{h}$ \\
$\lambda_{8}$ & $1.95 \mathrm{e}-8 / \mathrm{h}$ \\
$m$ & $4 \mathrm{~S} . \mathrm{U}$. \\
$a_{0}$ & $3.6 \mathrm{~mm}$ \\
$a_{c}$ & $9.3 \mathrm{~mm}$ \\
$C$ & $1.8 \mathrm{e}-12 \mathrm{~S} . \mathrm{U}$. \\
$f(R)_{\text {Max }}$ & $2 \mathrm{~S} . \mathrm{U}$. \\
$Y_{\text {Max }}$ & $1.18 \mathrm{~S} . \mathrm{U}$. \\
$\Delta \sigma_{\text {Max }}$ & $0 \mathrm{MPa}$ \\
$d_{c}$ & $10 / \mathrm{yr}$ \\
$P$ & $41 \mathrm{~b}$ \\
$D_{0}$ & $273 \mathrm{~mm}$ \\
$S$ & $101 \mathrm{Mpa}$ \\
$y$ & $0.4 \mathrm{~S} . \mathrm{U}$. \\
$d_{m}$ & $7 \mathrm{~mm} / \mathrm{yr}$ \\
$\lambda_{1}^{\prime}$ & $9.31 \mathrm{e}-8 / \mathrm{h}$ \\
$\lambda_{2}^{\prime}$ & $2.52 \mathrm{e}-6 / \mathrm{h}$ \\
$\lambda_{3}^{\prime}$ & $6.16 \mathrm{e}-7 / \mathrm{h}$ \\
$\lambda_{4}^{\prime}$ & $1.82 \mathrm{e}-5 / \mathrm{h}$ \\
$d_{c}{ }^{\prime}$ & $15 / \mathrm{yr}$ \\
\hline \hline
\end{tabular}



Fig. 13. BDD corresponding to the fault tree shown in Fig. 8.

degradation model. The PDMP related to the last group is presented as follows:

$$
\begin{aligned}
\overrightarrow{Z_{1,5}}(t) & =\left(\begin{array}{c}
N(t) \\
Y_{K_{1}}(t) \\
Y_{K_{2}}(t) \\
Y_{K_{3}}(t) \\
Y_{K_{4}}(t) \\
Y_{K_{5}}(t)
\end{array}\right) \in \boldsymbol{E}_{1,5} \\
& =\mathbb{R} \times S_{\boldsymbol{K}_{1}} \times \cdots \times \boldsymbol{S}_{\boldsymbol{K}_{5}}, \forall t \geq 0
\end{aligned}
$$

where $N(t)$ denotes the number of solicitations applied till $t$, $\dot{N}(t)=\left\{\begin{array}{ll}d_{c}, & \text { if } Y_{K_{5}}(t)=2_{K_{5}} \\ d_{c}^{\prime}, & \text { if } Y_{K_{5}}(t)=1_{K_{5}}\end{array}\right.$ and $Y_{K_{q}}(t), q=1,2, \ldots, 5$ are characterized by the related transition rates. 




Fig. 14. Estimated system reliability with/without dependency.

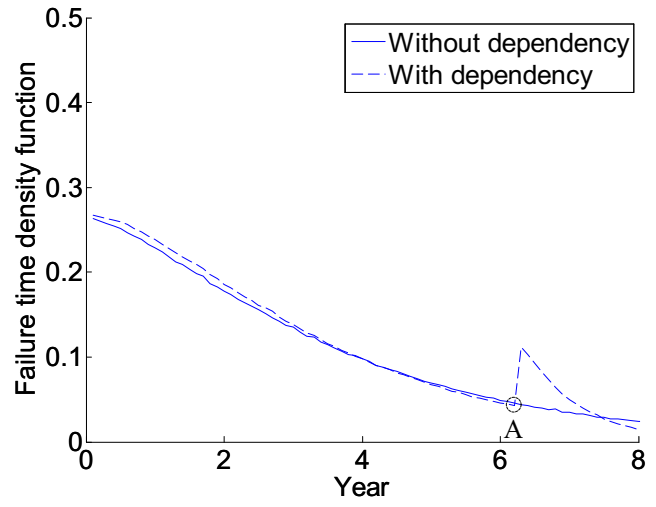

Fig. 15. System failure time density function with/without dependency.

MCS over a time horizon of eight years has been run $10^{6}$ times to solve the PDMPs and, then, estimate the probability of occurrence of each path. The numerical experiments are carried out in MATLAB on a personal computer with an Intel Core 2 Duo $\mathrm{CPU}$ at $3.06 \mathrm{GHz}$ and a RAM of 3.07 GB. The estimated system reliability with and without dependency throughout the time horizon, under accelerated conditions, is shown in Fig. 14. The average computation time is $34.3 \mathrm{~s}$. We can see from the figure that neglecting dependency can lead to overestimation of the system reliability. The system reliability with dependency has experienced one rapid decrease after around 6.2 years (point A), which is due to the valve failure in some simulation trials caused by the vibration of the pump. This sharp decrease in system reliability relates to the sharp increase in the system failure time density function, as shown in Fig. 15.

\section{CONClusion}

In this paper, we have proposed a framework for the reliability assessment of systems whose components have dependent competing degradation processes. The modeling framework rests on MSMs and PBMs, and the PDMP modeling approach is employed to treat dependencies between the degradation processes within one component or/and among components. The numerical solution involves the translation of the system fault tree into a BDD, and the estimation of the probabilities of the paths of events occurrences by MCS. The case study demonstrates the relevance of degradation process dependencies for the system reliability.

It is interesting to include failure isolation as future research in our proposed model. Failure detection and isolation can be used to mitigate degradation dependency by performing corresponding maintenance tasks or failure isolation actions.

\section{ACKNOWLEDGMENT}

The authors would like to thank D. Vasseur, A. Despujols, and E. Ardillon from the Department Industrial Risks Management, Electricité de France Research and Development for their insightful comments.

\section{REFERENCES}

[1] N. Gebraeel, A. Elwany, and J. Pan, "Residual life predictions in the absence of prior degradation knowledge," IEEE Trans. Rel., vol. 58, no. 1, pp. 106-117, Mar. 2009.

[2] J. Lawless and M. Crowder, "Covariates and random effects in a gamma process model with application to degradation and failure," Lifetime Data Anal., vol. 10, no. 3, pp. 213-227, 2004.

[3] M. Black, A. T. Brint, and J. R. Brailsford, "A semi-Markov approach for modelling asset deterioration," J. Oper. Res. Soc., vol. 56, no. 11, pp. 1241-1249, 2005.

[4] M. Chookah, M. Nuhi, and M. Modarres, "A probabilistic physics-offailure model for prognostic health management of structures subject to pitting and corrosion-fatigue," Rel. Eng. Syst. Safety, vol. 96, no. 12, pp. 1601-1610, 2011.

[5] M. J. Daigle and K. Goebel, "A model-based prognostics approach applied to pneumatic valves," Int. J. Progn. Health Manage., vol. 2, pp. 1-16, Aug. 2011.

[6] S. Reggiani et al., "Physics-based analytical model for HCS degradation in STI-LDMOS transistors," IEEE Trans. Electron Devices, vol. 58, no. 9, pp. 3072-3080, Sep. 2011.

[7] E. Keedy and Q. Feng, "A physics-of-failure based reliability and maintenance modeling framework for stent deployment and operation," Rel. Eng. Syst. Safety, vol. 103, pp. 94-101, Jul. 2012.

[8] R. Moghaddass and M. J. Zuo, "Multistate degradation and supervised estimation methods for a condition-monitored device," IIE Trans., vol. 46, no. 2, pp. 131-148, 2014.

[9] A. Lisnianski and G. Levitin, Multi-State System Reliability: Assessment, Optimization and Applications, vol. 6. Singapore: World scientific, 2003.

[10] W. Li and H. Pham, "Reliability modeling of multi-state degraded systems with multi-competing failures and random shocks," IEEE Trans. Rel., vol. 54, no. 2, pp. 297-303, Jun. 2005.

[11] M. Giorgio, M. Guida, and G. Pulcini, "An age- and state-dependent Markov model for degradation processes," IIE Trans., vol. 43, no. 9, pp. 621-632, 2011.

[12] Y.-H. Lin, Y.-F. Li, and E. Zio, "Integrating random shocks into multistate physics models of degradation processes for component reliability assessment," IEEE Trans. Rel., vol. 64, no. 1, pp. 154-166, Mar. 2015.

[13] L. Jiang, Q. Feng, and D. W. Coit, "Reliability and maintenance modeling for dependent competing failure processes with shifting failure thresholds," IEEE Trans. Rel., vol. 61, no. 4, pp. 932-948, Dec. 2012.

[14] N. Rasmekomen and A. K. Parlikad, "Maintenance optimization for asset systems with dependent performance degradation," IEEE Trans. Rel., vol. 62, no. 2, pp. 362-367, Jun. 2013.

[15] L. Xing, C. Wang, and G. Levitin, "Competing failure analysis in nonrepairable binary systems subject to functional dependence," Proc. Ins. Mech. Eng. O, J. Risk Rel., vol. 226, no. 4, pp. 406-416, 2012.

[16] C. Wang, L. Xing, and G. Levitin, "Competing failure analysis in phased-mission systems with functional dependence in one of phases," Rel. Eng. Syst. Safety, vol. 108, pp. 90-99, Dec. 2012.

[17] L. Xing and G. Levitin, "Combinatorial analysis of systems with competing failures subject to failure isolation and propagation effects," Rel. Eng. Syst. Safety, vol. 95, no. 1, pp. 1210-1215, 2010.

[18] C. Wang, L. Xing, and G. Levitin, "Reliability analysis of multi-trigger binary systems subject to competing failures," Rel. Eng. Syst. Safety, vol. 111, pp. 9-17, Mar. 2013.

[19] Y.-H. Lin, Y.-F. Li, and E. Zio, "Fuzzy reliability assessment of systems with multiple-dependent competing degradation processes," IEEE Trans. Fuzzy Syst., vol. 23, no. 5, pp. 1428-1438, Oct. 2015. 
[20] S. Song, D. W. Coit, and Q. Feng, "Reliability for systems of degrading components with distinct component shock sets," Rel. Eng. Syst. Safety, vol. 132, pp. 115-124, Dec. 2014.

[21] W. S. Lee, D. L. Grosh, F. A. Tillman, and C. H. Lie, "Fault tree analysis, methods, and applications-A review," IEEE Trans. Rel., vol. R-34, no. 3, pp. 194-203, Aug. 1985.

[22] R. M. Sinnamon and J. D. Andrews, "New approaches to evaluating fault trees," Rel. Eng. Syst. Safety, vol. 58, no. 2, pp. 89-96, 1997.

[23] C. Wang, L. Xing, and G. Levitin, "Explicit and implicit methods for probabilistic common-cause failure analysis," Rel. Eng. Syst. Safety, vol. 131, pp. 175-184, Nov. 2014.

[24] J. K. Vaurio, "An implicit method for incorporating common-cause failures in system analysis," IEEE Trans. Rel., vol. 47, no. 2, pp. 173-180, Jun. 1998

[25] J. K. Vaurio, "Treatment of general dependencies in system fault-tree and risk analysis," IEEE Trans. Rel., vol. 51, no. 3, pp. 278-287, Sep. 2002.

[26] L. Xing, G. Levitin, C. Wang, and Y. Dai, "Reliability of systems subject to failures with dependent propagation effect," IEEE Trans. Syst., Man, Cybern., Syst., vol. 43, no. 2, pp. 277-290, Mar. 2013.

[27] Y. Mo, L. Xing, and S. V. Amari, "A multiple-valued decision diagram based method for efficient reliability analysis of non-repairable phased-mission systems," IEEE Trans. Rel., vol. 63, no. 1, pp. 320-330, Mar. 2014.

[28] Y. Mo, L. Xing, and J. B. Dugan, "MDD-based method for efficient analysis on phased-mission systems with multimode failures," IEEE Trans. Syst., Man, Cybern., Syst., vol. 44, no. 6, pp. 757-769, Jun. 2014.

[29] S. Song, D. W. Coit, Q. Feng, and H. Peng, "Reliability analysis for multi-component systems subject to multiple dependent competing failure processes," IEEE Trans. Rel., vol. 63, no. 1, pp. 331-345, Mar. 2014.

[30] R. E. Bryant, "Graph-based algorithms for Boolean function manipulation," IEEE Trans. Comput., vol. C-35, no. 8, pp. 677-691, Aug. 1986.

[31] E. Zio, The Monte Carlo Simulation Method for System Reliability and Risk Analysis. London, U.K.: Springer, 2013.

[32] A. Rauzy, "New algorithms for fault trees analysis," Rel. Eng. Syst. Safety, vol. 40, no. 3, pp. 203-211, 1993.

[33] Z. Tang and J. B. Dugan, "BDD-based reliability analysis of phasedmission systems with multimode failures," IEEE Trans. Rel., vol. 55, no. 2, pp. 350-360, Jun. 2006

[34] L. Xing, "An efficient binary-decision-diagram-based approach for network reliability and sensitivity analysis," IEEE Trans. Syst., Man, Cybern. A, Syst., Humans, vol. 38, no. 1, pp. 105-115, Jan. 2008.

[35] M. Marseguerra and E. Zio, "Monte Carlo approach to PSA for dynamic process systems," Rel. Eng. Syst. Safety, vol. 52, no. 3, pp. 227-241, 1996.

[36] B. Bollig and I. Wegener, "Improving the variable ordering of OBDDs is NP-complete," IEEE Trans. Comput., vol. 45, no. 9, pp. 993-1002, Sep. 1996.

[37] S. J. Friedman and K. J. Supowit, "Finding the optimal variable ordering for binary decision diagrams," in Proc. 24th ACM/IEEE Design Autom. Conf., Miami Beach, FL, USA, 1987, pp. 348-356.

[38] S. Minato, N. Ishiura, and S. Yajima, "Shared binary decision diagram with attributed edges for efficient Boolean function manipulation," in Proc. 27th ACM/IEEE Design Autom. Conf., Orlando, FL, USA, 1990, pp. 52-57.

[39] M. Bouissou, F. Bruyère, and A. Rauzy, "BDD based fault-tree processing: A comparison of variable ordering heuristics," in Proc. Eur. Safety Rel. Assoc. Conf. (ESREL), vol. 3. Lisbon, Portugal, 1997, pp. 2045-2052.

[40] Y. Mo, F. Zhong, H. Liu, Q. Yang, and G. Cui, "Efficient ordering heuristics in binary decision diagram-based fault tree analysis," Qual. Rel. Eng. Int., vol. 29, no. 3, pp. 307-315, 2013.

[41] R. Tarjan, "Depth-first search and linear graph algorithms," Soc. Ind. Appl. Math. J. Comput., vol. 1, no. 2, pp. 146-160, 1972.

[42] K. S. Brace, R. L. Rudell, and R. E. Bryant, "Efficient implementation of a BDD package," in Proc. 27th ACM/IEEE Design Autom. Conf., Orlando, FL, USA, 1991, pp. 40-45.

[43] N. Limnios, Fault Trees, vol. 675. Hoboken, NJ, USA: Wiley, 2010.

[44] C. Cocozza-Thivent. (2011). Processus De Renouvellement Markovien Processus De Markov Déterministes Par Morceaux. [Online]. Available: http://perso-math.univ-mlv.fr/users/cocozza.christiane/recherchepageperso/PresentationRMetPDMP.html

[45] E. E. Lewis and F. Böhm, "Monte Carlo simulation of Markov unreliability models," Nuclear Eng. Design, vol. 77, no. 1, pp. 49-62, 1984.

[46] R. Coudray and J. M. Mattei, "System reliability: An example of nuclear reactor system analysis," Rel. Eng., vol. 7, no. 2, pp. 89-121, 1984
[47] S. Zhang, M. Hodkiewicz, L. Ma, and J. Mathew, "Machinery condition prognosis using multivariate analysis," in Engineering Asset Management. London, U.K.: Springer, 2006, pp. 847-854.

[48] P. Moussou et al., "Vibration investigation of a French PWR power plant piping system caused by cavitating butterfly valves," in Proc. ASME Publications PVP, vol. 420. Atlanta, GA, USA, 2001, pp. 99-106.

[49] P. Paris and F. Erdogan, "A critical analysis of crack propagation laws," J. Fluids Eng., vol. 85, no. 4, pp. 528-533, 1963.

[50] S. Marie et al., "French RSE-M and RCC-MR code appendices for flaw analysis: Presentation of the fracture parameters calculation-Part III: Cracked pipes," Int. J. Press. Vess. Piping, vol. 84, nos. 10-11, pp. 614-658, 2007.



Yan-Hui Lin received the B.Sc. degree in applied mathematics from Beihang University, Beijing, China, in 2010, the M.Sc. degree in applied mathematics from École Centrale Paris, Châtenay-Malabry, France, in 2010, and the M.Sc. degree in aircraft design from Beihang University in 2013. He is currently pursuing the Ph.D. degree with Chair System Science and the Energy Challenge, Fondation Electricité de France, CentraleSupélec, Université Paris-Saclay, Châtenay-Malabry.

His current research interests include reliability, degradation and maintenance modeling, Monte Carlo simulation, and optimization under uncertainty.

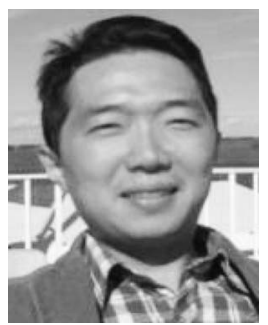

Yan-Fu Li (SM'14) received the Ph.D. degree in industrial engineering from the National University of Singapore, Singapore, in 2009.

$\mathrm{He}$ is an Assistant Professor with Chair System Science and the Energy Challenge, Fondation Electricité de France, CentraleSupélec, Université Paris-Saclay, Châtenay-Malabry, France. He was a Research Associate with the University of Tennessee, Knoxville, TN, USA. He has authored over 30 publications, all in refereed international journals, conferences, and books. His current research interests include reliability modeling, uncertainty analysis, evolutionary computing, and Monte Carlo simulation.

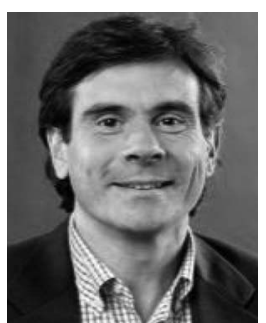

Enrico Zio (SM'09) received the Ph.D. degree in nuclear engineering from Politecnico di Milano, Milan, Italy, and the Massachusetts Institute of Technology, Cambridge, MA, USA, in 1995 and 1998, respectively.

$\mathrm{He}$ is currently the Director of the Chair System Science and the Energy Challenge, Fondation Electricité de France, CentraleSupélec, Université Paris-Saclay, Châtenay-Malabry, France, and a Full Professor with the Politecnico di Milano. His current research interests include characterization and modeling of the failure/repair/maintenance behavior of components, complex systems and their reliability, maintainability, prognostics, safety, vulnerability and security, Monte Carlo simulation methods, soft computing techniques, and optimization heuristics. 